Research Article; Received: March 17, 2021; Accepted: June 5, 2021

\title{
A GENERALIZATION OF PURELY EXTENDING MODULES RELATIVE TO A TORSION THEORY
}

\author{
Semra DOĞRUÖZ and Azime TARHAN \\ Adnan Menderes University, Aydın, TURKEY
}

\begin{abstract}
In this work we introduce a new concept, namely, purely $\tau_{s}$-extending modules (rings) which is torsion-theoretic analogues of extending modules and then we extend many results from extending modules to this new concept. For instance, we show that for any ring $R$ with unit, ${ }_{R} R$ is purely $\tau_{s}$-extending if and only if every cyclic $\tau$-nonsingular $R$-module is flat. Also, we make a classification for the direct sums of the rings to be purely $\tau_{s}$-extending.
\end{abstract}

\section{INTRODUCTION}

Injective modules have been intensively studied in the 1960s and 1970s in module theory and more generally in algebra. As a generalization of injective modules, extending modules (CS), that is every closed submodule is a direct summand, have been studied widely in last three decades. In general setting, Chatters and Hajarnavis 7], Harmancı and Smith 23], Kamal and Muller 24] and their schools can be mentioned involving studies of extending modules.

Recently, torsion-theoretic analogues of extending modules has been studied on many results and concepts, such primarily studies as, Asgari and Haghany 4], Berktaş, Doğruöz and Tarhan [6, Crivei [11, Çeken and Alkan 12], Doğruöz [13]. Clark 8 defined a module $M$ is purely extending if every submodule of $M$ is essential in a pure submodule of $M$, equivalently every closed submodule of $M$ is pure in $M$. A submodule $K$ of a module $M$ is essential (in $M$ ) if $N \cap K \neq$ 0 for every non-zero submodule $K$ of $M$. A submodule $K$ of a module $M$ is closed (in $M$ ) if $K$ has no proper essential extension in $M$, i.e., whenever $L$ is a submodule of $M$ such that $K$ is essential in $L$, then $K=L$. Al-Bahrani 1] generalized purely extending modules as a purely $y$-extending module using

2020 Mathematics Subject Classification. Primary 16S90, 16D40; Secondary 16E60.

Keywords. Pure submodule, closed submodule, (non)singular module, extending module, torsion theory.

@ sdogruoz@adu.edu.tr-Corresponding author; a.tarhan89@hotmail.com

(D) 0000-0002-7928-301X; 0000-0002-5363-1936. 
$s$-closed submodules which was defined by Goodearl 21 such as a submodule $N$ of a module $M$ is $s$-closed in $M$ if $M / N$ is nonsingular. So a module $M$ is called purely $y$-extending if every $s$-closed submodule of $M$ is pure in $M$. In fact, Al-Bahrani [1] belike misused the terminology of $s$-closed submodules. They used the term $y$-closed (purely $y$-extending) instead of $s$-closed (purely $s$-extending) respectively. In this study, we use $s$-closed submodule and purely $s$-extending module instead of $y$-closed submodule and purely $y$-extending module in the sense of Al-Bahrani 1 .

We use the concept 'purity' in the sense of Cohn [10 (as in [8]) which implies definition of Anderson and Fuller [3], that is, a submodule $N$ of an $R$-module $M$ is called pure submodule in $M$ in case $I N=N \cap I M$ for each finitely generated right ideal $I$ of the ring $R$ (see also [26] ). In the present paper we introduce purely $\tau_{s}$-extending modules and then we extend many results from [1], 8] and $[21$ to this new concept.

For instance, we show that:

Theorem 1; Let $R$ be a $\tau$-torsion ring and $M$ be an $R$-module. Let $E(M)$ be an injective hull of $M$. Then $M$ is a purely $\tau_{s}$-extending module if and only if $A \cap M$ is pure in $M$ for every direct summand $A$ of $E(M)$ such that the submodule $A \cap M$ is $\tau_{s}$-closed in $M$.

Proposition 5 Let $R$ be a ring with identity. Then ${ }_{R} R$ is purely $\tau_{s}$-extending if and only if every cyclic $\tau$-nonsingular $R$-module is flat.

and

Theorem 6 6 : Let $R$ be a commutative domain and every essential ideal of $R$ is $\tau$-dense in $R$. Then the following properties are equivalent:

(1): $R$ is a semi-hereditary ring.

(2): $R \oplus R$ is an extending module.

(3): $R \oplus R$ is a purely extending module.

(4): $R \oplus R$ is a purely $s$-extending module.

(5): $R \oplus R$ is a purely $\tau_{s}$-extending module.

(6): for each $n \in \mathbb{N}, \bigoplus_{n} R$ is an extending module.

(7): for each $n \in \mathbb{N}, \bigoplus_{n} R$ is a purely extending module.

(8): for each $n \in \mathbb{N}, \bigoplus_{n} R$ is a purely s-extending module.

(9): for each $n \in \mathbb{N}, \bigoplus_{n} R$ is a purely $\tau_{s}$-extending module.

which is a torsion-theoretic analogue of 8 , Proposition 1.6].

Throughout the work $R$ will be an associative ring with identity and all $R$-modules will be unitary left $R$-modules unless otherwise stated. $R$-Mod will be the category of unitary left $R$-modules, and all modules and module homomorphisms will belong to $R$-Mod. By a class $\mathcal{X}$ of $R$-modules we mean a collection of $R$-modules containing the zero module and closed under isomorphism, i.e., any module which is isomorphic to some module in $\mathcal{X}$ also belongs to $\mathcal{X}$. If a submodule $N$ of a module $M$ belongs to $\mathcal{X}$ class, then $N$ is called $\mathcal{X}$-submodule of $M$. The class of $\mathcal{X}$ closed under extension 
by short exact sequence we mean for a short exact sequence

$$
\mathrm{O} \longrightarrow \mathrm{C} \longrightarrow \mathrm{B} \longrightarrow \mathrm{A} \longrightarrow 0
$$

of $R$-modules $A, B, C$, if $A$ and $C$ are bought belong to the class of $\mathcal{X}$, then $B$ is also belongs to $\mathcal{X}$ class.

Let $\tau:=(\mathcal{T}, \mathcal{F})$ be a hereditary torsion theory on $R$-Mod. The modules in $\mathcal{T}$ are called $\tau$-torsion modules and the modules in $\mathcal{F}$ are called $\tau$-torsion-free modules. Let $M \in R$-Mod. Then the $\tau$-torsion submodule of $M$, denoted by $\tau(M)$, is defined to be the sum of all $\tau$-torsion submodules of $M$. Thus $\tau(M)$ is the unique largest $\tau$-torsion submodule of $M$ and $\tau(M / \tau(M))=0$ for an $R$-module $M$. Also the module $M$ is $\tau$-torsion (resp. $\tau$-torsion-free) if and only if $\tau(M)=M$ (resp. $\tau(M)=0$ ). In our study, we mean a ring $R$ is $\tau$-torsion if ${ }_{R} R$ is $\tau$-torsion.

Let $M$ be an $R$-module. A submodule $N$ of $M$ is called $\tau$-dense in $M$ if $M / N$ is $\tau$-torsion. A submodule $N$ of $M$ is called $\tau$-essential in $M$ denoted by $\left(N \leq_{\tau_{e}} M\right)$ if $N$ is essential in $M$ and $M / N$ is $\tau$-torsion (see [19], originally defined by Tsai in

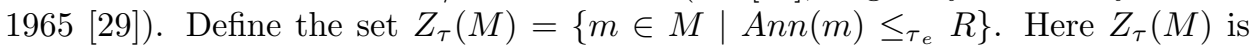
called the $\tau$-singular submodule of $M$. Then the module $M$ is called $\tau$-singular if $Z_{\tau}(M)=M$ and $\tau$-nonsingular if $Z_{\tau}(M)=0([20])$. We mean $Z(M)$ the singular submodule of a module $M$ which is consists of singular elements of $M$, i.e., elements annihilated by essential left ideals. The module $M$ is singular (resp. nonsingular) if $Z(M)=M$ (resp. $Z(M)=0$ ). For the singular and nonsingular notions (see also 21], 22] ). If a ring $R$ is $\tau$-torsion, then every left ideal $I$ of $R$ is $\tau$-dense in it, i.e., $R / I$ is $\tau$-torsion in the sense of 19$]$. Therefore, clearly $Z_{\tau}(M)=Z(M)$ over a $\tau$-torsion ring $R$.

For elementary, additional and unexplained terminology the reader is referred to 3 or 30 for module and ring theory, [19 and 28] for torsion theory, 15] for extending modules and $[26$ for homological algebra.

\section{Purely $\tau_{s}$-Extending Modules}

Definition 1. Let $M$ be an $R$-module and $N$ be a submodule of $M$. We call $N$ is a $\tau_{s}$-closed submodule of $M$ if the factor module $M / N$ is $\tau$-nonsingular and it is denoted by $N \leq \tau_{s} c M$.

Definition 2. Let $M$ be an $R$-module. We call $M$ is a purely $\tau_{s}$-extending module if every $\tau_{s}$-closed submodule of $M$ is pure in $M$.

Lemma 1. Let $R$ be a $\tau$-torsion ring. Then every $\tau_{s}$-closed submodule of a module $M$ is closed in $M$.

Proof. Let $N$ be a $\tau_{s}$-closed submodule of $M$. Then the factor module $M / N$ is $\tau$-nonsingular i.e., $Z_{\tau}(M / N)=0$. Since $R$ is $\tau$-torsion, clearly $Z_{\tau}(M / N)=$ $Z(M / N)$. Assume $N$ is not closed in $M$. Then there exists a submodule $K$ of $M$ such that $K$ contains $N$ as an essential submodule. So the factor module $K / N$ 
is singular 21. Hence $Z(K / N)=K / N$. On the other hand, $Z(K / N)=0$ since $Z(K / N)$ is a submodule of $Z(M / N)$. Hence $K / N$ is nonsingular. But since $K / N$ is singular, it must be zero (i.e $K / N=0$ ). Therefore, $N=K$ and so $N$ is closed submodule of $M$.

Corollary 1. Let $R$ be a $\tau$-torsion ring. Then every purely extending $R$-module is purely $\tau_{s}$-extending.

Proof. Let $M$ be a purely extending module and $\mathrm{N}$ be a $\tau_{s}$-closed submodule of $M$. Since $R$ is $\tau$-torsion $N$ is closed in $M$ by Lemma 11. From 8, Lemma 1.1] every closed submodule of $M$ is pure in $M$. So $N$ is pure in $M$. Therefore $M$ is purely $\tau_{s}$-extending module.

As in general extending module theory we have some of the fundamental properties of purely $\tau_{s}$-extending modules as follows:

Lemma 2. Let $M=M_{1} \oplus M_{2}$ be a purely $\tau_{s}$-extending module. Then $M_{1}$ and $M_{2}$ are also purely $\tau_{s}$-extending modules i.e., any direct summand of a purely $\tau_{s}$-extending module is purely $\tau_{s}$-extending.

Proof. Let $M=M_{1} \oplus M_{2}$ be a purely $\tau_{s}$-extending module and let $N_{1}$ be a $\tau_{s}$-closed submodule of $M_{1}$. Then $Z_{\tau}\left(M_{1} / N_{1}\right)=0$. For the proof we want to show that $N_{1}$ is pure in $M_{1}$. First let us show that $N_{1}$ is $\tau_{s}$-closed in $M$ i.e., $\left(M / N_{1}\right)$ is $\tau$-nonsingular.

Assume $M / N_{1}$ is not $\tau$-nonsingular module. Thus $Z_{\tau}\left(M / N_{1}\right) \neq 0$. Then there exists an element $N_{1} \neq m+N_{1} \in M / N_{1}$ such that $\operatorname{Ann}\left(m+N_{1}\right) \leq_{\tau_{e}} R$. On the other hand, since $m \in M=M_{1} \oplus M_{2}$, there exist $m_{1} \in M_{1}$ and $m_{2} \in M_{2}$ such that $m=m_{1}+m_{2}$ and this writing unique. Thus

$$
\begin{aligned}
\operatorname{Ann}\left(m+N_{1}\right) & =\operatorname{Ann}\left(\left(m_{1}+m_{2}\right)+N_{1}\right)=\operatorname{Ann}\left(m_{1}+N_{1}+m_{2}+N_{1}\right) \\
& =\operatorname{Ann}\left(m_{1}+N_{1}\right) \cap \operatorname{Ann}\left(m_{2}+N_{1}\right)
\end{aligned}
$$

(see 3. Proposition 2.16]). In addition, since $\operatorname{Ann}\left(m+N_{1}\right) \leq_{\tau_{e}} R$, we have $\operatorname{Ann}\left(m_{1}+N_{1}\right) \cap \operatorname{Ann}\left(m_{2}+N_{1}\right) \leq_{\tau_{e}} R$. Since Ann $\left(m_{1}+N_{1}\right) \cap \operatorname{Ann}\left(m_{2}+N_{1}\right) \subseteq$ $\operatorname{Ann}\left(m_{1}+N_{1}\right) \subseteq R$, we have $\operatorname{Ann}\left(m_{1}+N_{1}\right) \leq_{\tau_{e}} R$. But this contradicts with $Z_{\tau}\left(M / N_{1}\right) \neq 0$. Hence $Z_{\tau}\left(M / N_{1}\right)=0$ i.e., $N_{1}$ is a $\tau_{s}$-closed submodule of $M$. By the hypothesis $N_{1}$ is pure in $M$ since $M$ is purely $\tau_{s}$-extending module. By 17 , Proposition $1.2(2)] N_{1}$ is pure in $M_{1}$. Thus $M_{1}$ is purely $\tau_{s}$-extending module. Similarly it can be shown that $M_{2}$ is also purely $\tau_{s}$-extending module.

Corollary 2. Let $M=\bigoplus_{i \in I} M_{i}$ be a purely $\tau_{s}$-extending module where $I$ is a finite index set. Then for every $i \in I, M_{i}$ is purely $\tau_{s}$-extending.

Proof. It is clear from Lemma 2 .

Lemma 3. Let $C$ be an $R$-module. Then $C$ is a $\tau$-nonsingular module if and only if $H_{o m}(A, C)=0$ for every $\tau$-singular R-module $A$. 
Proof. Let $f: A \longrightarrow C$ be an $R$-module homomorphism where $C$ is a $\tau$-nonsingular module and $A$ is a $\tau$-singular $R$-module. Then $f(A)=f\left(Z_{\tau}(A)\right)$. We show $f\left(Z_{\tau}(A)\right) \leq Z_{\tau}(C)$. If $x \in f\left(Z_{\tau}(A)\right)$ then there is an element $a \in Z_{\tau}(A)$ such that $x=f(a)$. So $\operatorname{Ann}(a) \leq_{\tau_{e}} R$. If $r \in \operatorname{Ann}(a)$, then $r x=r f(a)=f(r a)=0$ i.e., $r \in \operatorname{Ann}(x)$. Since $A n n(a) \leq A n n(x) \leq R$, we have $A n n(x) \leq_{\tau_{e}} R$ i.e., $x \in Z_{\tau}(C)$. By the hypothesis, since $Z_{\tau}(C)=0, f=0$ and thus $\operatorname{Hom}_{R}(A, C)=0$.

For the converse let $H_{0}(A, C)=0$ for every $\tau$-nonsingular $R$-module $A$. Specially $\operatorname{Hom}_{R}\left(Z_{\tau}(C), C\right)=0$. So the inclusion map $Z_{\tau}(C) \longrightarrow C$ is zero. Hence $Z_{\tau}(C)=0$ and so $C$ is $\tau$-nonsingular module.

Lemma 4. The class of $\tau$-nonsingular modules is closed under extensions by short exact sequences.

Proof. Let $C$ and $A$ be $\tau$-nonsingular modules and consider the following short exact sequence

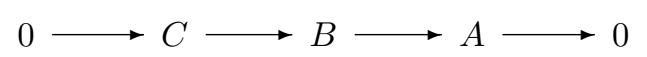

For every $\tau$-singular $R$-module $M$, using Lemma 3 , we have $\operatorname{Hom}_{R}(M, C)=0$ and $\operatorname{Hom}_{R}(M, A)=0$. Then the following short exact sequence

$$
0 \longrightarrow \operatorname{Hom}_{R}(M, C) \longrightarrow \operatorname{Hom}_{R}(M, B) \longrightarrow \operatorname{Hom}_{R}(M, A) \longrightarrow 0
$$

yields $H_{R}(M, B)=0$. Again by Lemma 3 the $R$-module $B$ must be $\tau$-nonsingular.

Next we can show $\tau_{s}$-closed submodules have transitivity property.

Lemma 5. Let $M$ be an R-module and let $K$ and $N$ be submodules of $M$ such that $K \leqslant N$. If $K$ is $\tau_{s}$-closed submodule of $N$ and $N$ is $\tau_{s}$-closed submodule of $M$, then $K$ is $\tau_{s}$-closed submodule of $M$.

Proof. Since $K$ is $\tau_{s}$-closed submodule of $N$ and $N$ is $\tau_{s}$-closed submodule of $M$, $Z_{\tau}(N / K)=0$ and $Z_{\tau}(M / N)=0$. We must show that $Z_{\tau}(M / K)=0$. Consider the following short exact sequence

$$
0 \longrightarrow \mathrm{N} / \mathrm{K} \longrightarrow \mathrm{M} / \mathrm{K} \longrightarrow \mathrm{M} / \mathrm{N} \longrightarrow 0
$$

By Lemma 4, the class of $\tau$-nonsingular modules are closed under extensions by short exact sequences. Since $N / K$ and $M / N$ are both $\tau$-nonsingular, $M / K$ is $\tau$-nonsingular. Hence $Z_{\tau}(M / K)=0$. Thus $K$ is $\tau_{s}$-closed submodule of $M$.

Now we have some basic properties as follows.

Lemma 6. Any $\tau_{s}$-closed submodule of a purely $\tau_{s}$-extending module is purely $\tau_{s}$-extending. 
Proof. Let $M$ be a purely $\tau_{s}$-extending module and let $N$ be a $\tau_{s}$-closed submodule of $M$. Then $M / N$ is $\tau$-nonsingular. Let $K$ be a $\tau_{s}$-closed submodule of $N$. Then by Lemma $5, K$ is a $\tau_{s}$-closed submodule of $M$. Since $M$ is purely $\tau_{s}$-extending module, $K$ is pure in $M$. By [17, Proposition $1.2(2)], K$ is pure in $N$. So $N$ is purely $\tau_{s}$-extending module.

There exist submodules $K, L$ of a module $M$ such that $K$ and $L$ both closed submodules of $M$ but $K \cap L$ is not closed in $K, L$ or $M$ (see 21, Example 1.6]). But we have the following in our case.

Proposition 1. Let $M$ be an $R$-module and $N, K$ be $\tau_{s}$-closed submodules of $M$. Then $N \cap K$ is a $\tau_{s}$-closed submodule of $M$.

Proof. Let $M$ be an $R$-module and $N, K$ be $\tau_{s}$-closed submodules of $M$. Then $M / K$ and $M / N$ are $\tau$-nonsingular, i.e., $Z_{\tau}(M / N)=0$ and $Z_{\tau}(M / K)=0$. Assume $Z_{\tau}(M /(N \cap K)) \neq 0$. Then there is a $(N \cap K) \neq \bar{m} \in M /(N \cap K)$ such that $\operatorname{Ann}(\bar{m}) \leq_{\tau_{e}} R$. Now for $\bar{m}=m+(N \cap K), m \notin N \cap K$. On the other hand for $m \in M$, choose the elements $\hat{m}=m+N \in M / N$ and $\tilde{m}=m+K \in M / K$. Then we have $\operatorname{Ann}(\bar{m}) \subseteq A n n(\hat{m})$ and $\operatorname{Ann}(\bar{m}) \subseteq A n n(\tilde{m})$. Indeed, now let $0 \neq r \in A n n(\bar{m})$. Then $r \bar{m}=0$ and so $r m+(N \cap K)=N \cap K$. Hence $r m \in N \cap K$. So we have $r m \in N$ and $r m \in K$. Thus $r m+N=N$ and $r m+K=K$, i.e. $r \hat{m}=0$ and $r \tilde{m}=0$. Consequently $r \in \operatorname{Ann}(\hat{m})$ and $r \in \operatorname{Ann}(\tilde{m})$. Hence $\operatorname{Ann}(\bar{m}) \subseteq$ $\operatorname{Ann}(\hat{m})$ and $\operatorname{Ann}(\bar{m}) \subseteq \operatorname{Ann}(\tilde{m})$. On the other hand, since $A n n(\bar{m}) \leq_{\tau_{e}} R$ we have $\operatorname{Ann}(\hat{m}) \leq_{\tau_{e}} R$ and $\operatorname{Ann}(\tilde{m}) \leq_{\tau_{e}} R$. Then by hypothesis $Z_{\tau}(M / N)=0$ and $Z_{\tau}(M / K)=0$, we have $m \in N$ and $m \in K$ and so $m \in N \cap K$. Hence $\bar{m}=m+(N \cap K)=N \cap K$. This is a contradiction. Thus $Z_{\tau}(M /(N \cap K))=0$. Therefore, $N \cap K$ is a $\tau_{s}$-closed submodule of $M$.

Corollary 3. Any intersection of $\tau_{s}$-closed submodules is also $\tau_{s}$-closed.

Proof. It is an evident result of Proposition 1.

Lemma 7. Let $M$ be an $R$-module and let $K, L$ be submodules of $M$ such that $K \leqslant L$. If $L$ is a $\tau_{s}$-closed submodule of $M$, then $L / K$ is a $\tau_{s}$-closed submodule of $M / K$.

Proof. Let $L$ be a $\tau_{s}$-closed submodule of $M$. Then $Z_{\tau}(M / L)=0$. On the other hand, $(M / K) /(L / K) \cong M / L$ and since $\tau$-nonsingular modules are closed under isomorphisms, $Z_{\tau}((M / K) /(L / K))=0$. Hence $L / K$ is $\tau_{s}$-closed in $M / K$.

Lemma 8. Let $M$ be an $R$-module and let $K, L$ be submodules of $M$ such that $K \leq$ $L$. If the submodule $L / K$ is $\tau_{s}$-closed in $M / K$, then $L$ is a $\tau_{s}$-closed submodule of $M$.

Proof. Since $L / K$ is a $\tau_{s}$-closed submodule of $M / K, Z_{\tau}((M / K) /(L / K))=0$. Since $(M / K) /(L / K) \cong M / L$ and $\tau$-nonsingular modules are closed under isomorphisms, $Z_{\tau}(M / L)=0$. Hence $L$ is a $\tau_{s}$-closed submodule of $M$. 
Proposition 2. Let $M$ be a purely $\tau_{s}$-extending $R$-module and $N$ be a $\tau_{s}$-closed submodule of $M$. Then the factor module $M / N$ is purely $\tau_{s}$-extending.

Proof. Let $M$ be a purely $\tau_{s}$-extending $R$-module and $N$ be a $\tau_{s}$-closed submodule of $M$. By the definition of purely $\tau_{s}$-extending module, $N$ is pure in $M$. For $N \leq K \leq M$ let $K / N$ be $\tau_{s}$-closed in $M / N$. Now $(M / N) /(K / N) \simeq M / K$ and since the $\tau$-nonsingular modules are closed under isomorphisms, $Z_{\tau}(M / K)=0$. So $K$ is $\tau_{s}$-closed submodule of $M$. Since $M$ is purely $\tau_{s}$-extending, $K$ is pure in $M$. By [17, Proposition $1.2(3)] K / N$ is pure in $M / N$. Thus $M / N$ is purely $\tau_{s}$-extending.

Let $M$ be an $R$-module. For an arbitrary submodule $N$ of $M$ by Zorn's Lemma there is a submodule $K$ of $M$ maximal with respect to $N$ is essential in $K$. The submodule $K$ is called closure of $N$ in $M([27)$. See also 14 for torsion theoretic version of closures.

Now we give another generalization of closures relative to a torsion theory as follows:

Definition 3. Let $M$ be an $R$-module and let $N$ be a submodule of $M$. The smallest $\tau_{s}$-closed submodule $K$ of $M$ which is containing $N$ is called $\tau_{s}$-closure of $N$ in $M$. The $\tau_{s}$-closure of $N$ is denoted by $N^{-\tau_{s}}$.

Lemma 9. Every submodule $N$ of an $R$-module $M$ has a $\tau_{s}$-closure in $M$.

Proof. Let $M$ be an $R$-module and $N$ be a submodule of $M$. Now define the set $\mathcal{S}=\left\{K \leq M \mid N \subseteq K\right.$ and $\left.K \leq_{\tau_{s} c} M\right\}$. Since $Z_{\tau}(M / M)=0, M$ is $\tau_{s^{-}}$closed in $M$ and so $M \in \mathcal{S}$. Then $\mathcal{S}$ is non-empty. Let $\mathcal{C}$ be a chain in $\mathcal{S}$. Take $C=\bigcap_{K_{i} \in \mathcal{C}} K_{i}$. By Corollary $3 C$ is a $\tau_{s}$-closed submodule of $M$. Then $C \in \mathcal{S}$. By Zorn's Lemma there is a minimal element in $\mathcal{S}$. If we call this element such as $H$ then $H$ is $\tau_{s}$-closure of $N$ in $M$. Thus every submodule $N$ of $M$ has a $\tau_{s}$-closure in $M$.

Proposition 3. An R-module $M$ is a purely $\tau_{s}$-extending if and only if the $\tau_{s}$-closure of $N$ (i.e., $N^{-\tau_{s}}$ ) is pure in $M$ for every submodule $N$ of $M$.

Proof. Let $M$ be a purely $\tau_{s}$-extending module. Then every $\tau_{s}$-closed submodule of $M$ is pure in $M$. By Zorn's Lemma every submodule $N$ of $M$ has a $\tau_{s}$-closure in $M$. By the definition of $\tau_{s}$-closure, the submodule $N^{-\tau_{s}}$ is $\tau_{s}$-closed in $M$ and by the hypothesis the submodule $N^{-\tau_{s}}$ is pure in $M$.

Conversely, let $K$ be a $\tau_{s}$-closed submodule in $M$. By the definition of $\tau_{s^{-}}$-closure, $K^{-\tau_{s}}=K$. By the hypothesis $K^{-\tau_{s}}$ i.e. $K$ is a pure submodule in $M$. Then any $\tau_{s}$-closed submodule of $M$ is pure in $M$. Thus $M$ is a purely $\tau_{s}$-extending module.

Theorem 1. Let $R$ be a $\tau$-torsion ring, let $M$ be an $R$-module and $E(M)$ be the injective hull of $M$. Then, $M$ is a purely $\tau_{s}$-extending module if and only if $A \cap M$ is pure in $M$ for every direct summand $A$ of $E(M)$ such that the submodule $A \cap M$ is $\tau_{s}$-closed in $M$. 
Proof. Let $R$ be a $\tau$-torsion ring, $M$ be an $R$-module, $E(M)$ be the injective hull of $M$ and $M$ be a purely $\tau_{s}$-extending module. Then for every direct summand $A$ of $E(M)$ such that $A \cap M$ is a $\tau_{s}$-closed submodule of $M$ it is clear that $A \cap M$ is pure in $M$.

Conversely, let $A$ be a $\tau_{s}$-closed submodule of $M$ and let $B$ be a complement of $A$ in $M$. Then $A \oplus B$ is essential in $M$ 21, Proposition 1.3]. Now it is clear that $A \oplus B$ is essential in $E(M)$. Hence $E(A) \oplus E(B)=E(A \oplus B)=E(M)$ 22. Since $A=A \cap M \leq_{e} E(A) \cap M,(E(A) \cap M) / A$ is singular (see 21]). Moreover, since $R$ is $\tau$-torsion ring $(E(A) \cap M) / A$ is $\tau$-singular. On the other hand since $(E(A) \cap M) / A \leq M / A$ and $A$ is $\tau_{s}$-closed submodule of $M, M / A$ is $\tau$-nonsingular and thus $(E(A) \cap M) / A$ is $\tau$-nonsingular. Therefore, $(E(A) \cap M) / A=0$ and so $E(A) \cap M=A$. Since $A$ is $\tau_{s}$-closed in $M, E(A) \cap M$ is also $\tau_{s}$-closed in $M$. Since $E(A)$ is a direct summand of $E(M)$ by the hypothesis $E(A) \cap M$ is a pure submodule of $M$. Hence $A$ is pure in $M$. Thus $M$ is a purely $\tau_{s}$-extending module.

Theorem 2. Let $R$ be a $\tau$-torsion ring, let $M$ be an $R$-module and let $E(M)$ be the injective hull of $M$. Assume $A+M$ be a flat module for every direct summand $A$ of $E(M)$ with $A \cap M$ is $\tau_{s}$-closed submodule of $M$. Then $M$ is a purely $\tau_{s}$-extending module.

Proof. Let $A$ be a direct summand of $E(M)$ such that $A \cap M$ is $\tau_{s}$-closed in $M$. Consider the following short exact sequences of $R$-modules

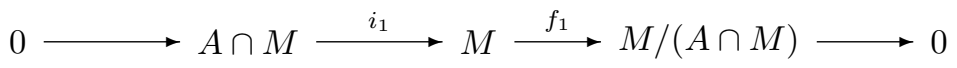

and

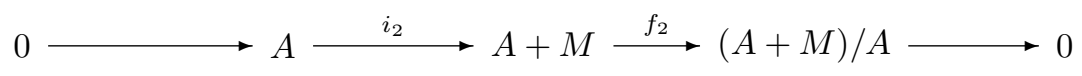

where $i_{1}, i_{2}$ are inclusion maps and $f_{1}, f_{2}$ are natural epimorphisms. Since $A$ is a direct summand of $E(M)$, there is a submodule $A^{\prime}$ of $E(M)$ such that $E(M)=$ $A \oplus A^{\prime}$. Thus $A$ is also a direct summand of $A+M$ such as $A+M=(A+M) \cap$ $E(M)=(A+M) \cap\left(A \oplus A^{\prime}\right)=A \oplus\left((A+M) \cap A^{\prime}\right)$. Here $\left((A+M) \cap A^{\prime}\right)$ is flat as a direct summand of a flat module $A+M$. Since $(A+M) / A \cong\left((A+M) \cap A^{\prime}\right)$, $(A+M) / A$ is flat. On the other hand, the factor module $M /(A \cap M)$ is again flat since $M /(A \cap M) \cong(A+M) / A$. By [17, Theorem 1.7] $A \cap M$ is pure in $M$. Hence by Theorem $1, M$ is a purely $\tau_{s}$-extending module. 


\section{Purely $\tau_{s}$-Extending Rings}

If the ring $R$ is purely $\tau_{s}$-extending as an $R$-module over itself then $R$ is called purely $\tau_{s}$-extending.

A (von Neumann ) regular ring $R$ as an $R$-module over itself, i.e., ${ }_{R} R$ can be given an example of purely $\tau_{s}$-extending ring since every left ideal is pure in it by 17 , Theorem 2.1].

Fieldhouse in 17] generalizing (von Neumann) regular ring and define, for any ring $R$, an $R$-module $M$ is called (von Neumann) regular if all its submodules are pure in $M$.

Therefore, since all (left) $R$-modules over a (von Neumann) regular ring is regular by [17, Theorem 3.1], thus all $R$-modules over a (von Neumann) regular ring $R$ is purely $\tau_{s}$-extending. Also any regular module over any ring $R$ can be given as an example of purely $\tau_{s}$-extending modules.

3.1. Multiplication Modules. Let $R$ be a commutative ring and $M$ be an $R$-module. For every submodule $N$ of $M$ if there exists an ideal $I$ of $R$ such that $N=I M$, then $M$ is called a multiplication module. For every submodule $N$ of $M$ let us define

$$
(N: M)=\{r \in R \mid r M \subseteq N\} .
$$

Then $M$ is an multiplication $R$-module if and only if $N=(N: M) M([5)$.

Definition 4. [9] Let $M$ be an $R$-module and $N$ be a submodule of $M$. If

$$
N=\operatorname{Hom}(M, N) N=\Sigma\{\varphi(N) \mid \varphi: M \rightarrow N\}
$$

then $N$ is called an idempotent submodule of $M$. If every submodule of $M$ is idempotent, then $M$ is called a fully idempotent module.

Theorem 3. [16, Teorem 2.11] Let $M$ be a multiplication $R$-module and $M=$ $M_{1} \oplus M_{2}$, is a direct sum of fully idempotent submodules $M_{1}$ and $M_{2}$. Then $M$ is a fully idempotent module.

Lemma 10. [16, Lemma 2.13] Let $M$ be a fully idempotent $R$-module, $N$ be a submodule of $M$ and $I$ be an ideal of $R$. Then $N \cap M I=N I$, i.e., $N$ is pure in $M$.

Now we can give the following teorem by using fully idempotent submodules:

Theorem 4. Let $R$ be a commutative ring and let $M=M_{1} \oplus M_{2}$ be a multiplication $R$-module with fully idempotent submodules $M_{1}, M_{2}$ of $M$. Then $M$ is a purely $\tau_{s}$-extending module.

Proof. Let $M$ be a multiplication $R$-module and $N$ be a $\tau_{s}$-closed submodule of $M$. By Teorem $3 M$ is fully idempotent $R$-module and by Lemma 10 the $\tau_{s}$-closed submodule $N$ of $M$ is pure in $M$. Hence $M$ is purely $\tau_{s}$-extending.

Now we can give a characterization of a purely $\tau_{s}$-extending $R$-module with a ring as follows: 
Proposition 4. Let $R$ be a commutative ring and let $M$ be a faithful multiplication $R$-module. If ${ }_{R} R$ is purely $\tau_{s}$-extending module then $M$ is also purely $\tau_{s}$-extending module.

Proof. Let $N$ be a $\tau_{s}$-closed submodule of $M$. Since $M$ is multiplication $R$-module, we can write $N=(N: M) M$. Claim: $(N: M)$ is $\tau_{s}$-closed submodule in ${ }_{R} R$. Assume $(N: M)$ is not $\tau_{s}$-closed in $R$. Then $R /(N: M)$ is not $\tau$-nonsingular that is, $Z_{\tau}(R /(N: M)) \neq 0$. Then there exists at least one non-zero element $\bar{r}$ of $R /(N: M)$ such that $\operatorname{Ann}(r+(N: M))$ is $\tau$-essential in $R$. So $\bar{r}=r+(N$ : $M) \neq(N: M)$. Then there is an element $0 \neq m_{0} \in M$ such that $r m_{0} \notin N$. Now $\operatorname{Ann}(r+(N: M)) \subseteq \operatorname{Ann}\left(r m_{0}+N\right)$. If $s \in \operatorname{Ann}(r+(N: M))$, then $s r+(N: M)=(N: M)$. Hence we have $s r \in(N: M)$ so it is easy to check that $(s r) M \subseteq N(*)$. Let us show that $s \in A n n\left(r m_{0}+N\right)$. Now $s\left(r m_{0}+N\right)=s r m_{0}+N$ but since $(s r) M \subseteq N$ and by (*) for $m_{0} \in M$, srm ${ }_{0} \in N$, i.e., $s r m_{0}+N=N$. So $s \in \operatorname{Ann}\left(r m_{0}+N\right)$. Hence we have $\operatorname{Ann}(r+(N: M)) \subseteq \operatorname{Ann}\left(r m_{0}+N\right)$. On the other hand, since $N$ is $\tau_{s}$-closed in $M$ it is clear that $M / N \tau$-nonsingular. So $r m_{0}+N=N$ but it contradicts with $r m_{0} \notin N$. Hence $(N: M)$ must be $\tau_{s}$-closed in $R$. Moreover since ${ }_{R} R$ is purely $\tau_{s}$-extending, $(N: M)$ is pure in $R$, i.e., $I(N: M)=I R \cap(N: M)$ for every finitely generated ideal $I$ of $R$. Thus $I(N: M)=I R \cap(N: M)=I \cap(N: M)$. Therefore, by $N=(N: M) M$ we write $I N=I(N: M) M=(I \cap(N: M)) M$. On the other hand, the equality $(I \cap(N: M)) M=I M \cap(N: M) M$ holds since $R$ is a commutative ring and $M$ is a faithful multiplication $R$-module by applying [2, Proposition 1.6 (i)].

Now for the finitely generated ideal $I$ of $R$, we have $I N=I(N: M) M=(I \cap(N: M)) M=I M \cap(N: M) M=I M \cap N([5])$. Therefore, the $\tau_{s}$-closed submodule $N$ of $M$ is pure in $M$. Hence $M$ is a purely $\tau_{s}$-extending module.

Remark 1. [26, Proposition 3.46] Let $R$ be an arbitrary ring. The left $R$-module $R$ is a flat left $R$-module.

In the sequel we use the flat ring in the sense of Rotman [26, Proposition 3.46], i.e the ring $R$ is flat if ${ }_{R} R$ is flat.

Proposition 5. Let $R$ be an arbitrary ring. Then ${ }_{R} R$ is purely $\tau_{s}$-extending if and only if every cyclic $\tau$-nonsingular $R$-module is flat.

Proof. Let $R R$ be a purely $\tau_{s}$-extending module. Let $M=R a$ be a cyclic $\tau$-nonsingular $R$-module which is generated by $a$. Define the map $f: R \rightarrow M$ with $f(r)=r a$. Clearly $f$ is an epimorphism and $\operatorname{Ker}(f)=A n n(a)$. So $R / \operatorname{Ker}(f)=R / A n n(a) \cong$ $R a$. Moreover, since $R a$ is a $\tau$-nonsingular module and the class of $\tau$-nonsingular modules is closed under isomorphisms $R / \operatorname{Ann}(a)$ is $\tau$-nonsingular. Hence $A n n(a)$ is $\tau_{s}$-closed in $R$. By the hypothesis $A n n(a)$ is pure in $R$. Since $R$ is flat and $A n n(a)$ is pure in $R, R / \operatorname{Ann}(a)$ is flat by [3, Lemma 19.18]. Therefore, $R a$ is flat. 
Conversely, let $K$ be a $\tau_{s}$-closed ideal of $R$. Then $R / K$ is $\tau$-nonsingular. By the hypothesis $R / K$ is flat as a left $R$-module. Thus by [3, Lemma 19.18], $K$ is pure in $R$. Thus ${ }_{R} R$ is a purely $\tau_{s}$-extending.

Theorem 5. Let $R$ be a ring. Then $R \oplus R$ is purely $\tau_{s}$-extending if and only if every $\tau$-nonsingular 2-generated $R$-module is flat.

Proof. Let $M=R m_{1}+R m_{2}$ be a $\tau$-nonsingular $R$-module. Define the map $f$ : $R \oplus R \rightarrow M$ with $f\left(r_{1}, r_{2}\right)=r_{1} m_{1}+r_{2} m_{2}$. Now it is clear that $f$ is an epimorphism. Hence $(R \oplus R) / \operatorname{Ker}(f) \cong M$. Since $(R \oplus R) / \operatorname{Ker}(f)$ is $\tau$-nonsingular, $\operatorname{Ker}(f)$ is a $\tau_{s}$-closed submodule of $R \oplus R$. By the hypothesis $\operatorname{Ker}(f)$ is pure in $R \oplus R$. Since $R$ is is flat as an $R$-module, $R \oplus R$ is flat ([21]). Thus by [17, Proposition $1.3(3)]$, we have the $R$-module $M$ is flat.

For the converse, let $C$ be a $\tau_{s}$-closed submodule of $R \oplus R$. Then $(R \oplus R) / C$ is $\tau$-nonsingular. On the other hand, since $R \oplus R$ is a 2 -generated $R$-module, $(R \oplus R) / C$ is also a 2-generated $\tau$-nonsingular $R$-module. By the hypothesis $(R \oplus R) / C$ is flat. Then by [17, Theorem 1.7] we get $C$ is pure in $R \oplus R$. Thus $R \oplus R$ is purely $\tau_{s^{-}}$ extending.

Corollary 4. Let $R$ be a ring and $I$ be a finite index set. Then $\oplus_{I} R$ is purely $\tau_{s}$-extending if and only if every $\tau$-nonsingular $I$-generated $R$-module is flat.

3.2. Semi-hereditary Rings. Let $R$ be a ring with unit element. If every left (right) ideal of $R$ is projective then $R$ is called a left (right) hereditary ring . If every finitely generated left (right) ideal of $R$ is projective then $R$ is called a left (right) semi-hereditary ring ( 28]). A module $M$ over a commutative domain $R$ is said to be torsion-free if for $m \in M$ and $r \in R, r m=0 \Rightarrow r=0$ or $m=0$ [25].

Now we can give the following generalized characterization of purely $\tau_{s}$-extending modules.

Theorem 6. Let $R$ be a commutative domain and every essential ideal of $R$ is $\tau$-dense in $R$. Then the following properties are equivalent:

(1): $R$ is a semi-hereditary ring.

(2): $R \oplus R$ is an extending module.

(3): $R \oplus R$ is a purely extending module.

(4): $R \oplus R$ is a purely s-extending module.

(5): $R \oplus R$ is a purely $\tau_{s}$-extending module.

(6): for each $n \in \mathbb{N}, \bigoplus_{n} R$ is an extending module.

(7): for each $n \in \mathbb{N}, \bigoplus_{n} R$ is a purely extending module.

(8): for each $n \in \mathbb{N}, \bigoplus_{n} R$ is a purely s-extending module.

(9): for each $n \in \mathbb{N}, \bigoplus_{n} R$ is a purely $\tau_{s}$-extending module.

Proof. The equivalence of (1), (2) and (6) are given in [15, Corollary 12.10].

In addition the equivalence of $(1),(2),(3),(6)$ and $(7)$ are given in 8 , Proposition 1.6]. 
$(3) \Leftrightarrow(4)$. Every $s$-closed submodule of a module $M$ is closed in $M$. But converse is true if $M$ is nonsingular [21, Proposition 2.4]. Here since $R$ is commutative domain, $R$ is nonsingular. Therefore, the notion of closed submodule and $s$-closed submodule coincide. Thus the proof is clear by [8, Lemma 1.1] in fact, Lemma 1.1 is originally given by Fuchs [18].

$(7) \Leftrightarrow(8)$. It can be easily checked be like $(3) \Leftrightarrow(4)$.

$(5) \Rightarrow(4)$. Let $K$ be a $s$-closed submodule of $R \oplus R$. Then $(R \oplus R) / K$ is nonsingular. Since any nonsingular module is $\tau$-nonsingular. $(R \oplus R) / K$ is a $\tau$-nonsingular. By the hypothesis $K$ is pure in $R \oplus R$. Hence $R \oplus R$ is a purely $s$-extending module.

The implication of $(9) \Rightarrow(8)$ is a generalization of $(5) \Rightarrow(4)$.

$(1) \Rightarrow(5)$. Let $K$ be a $\tau_{s}$-closed submodule of $R \oplus R$. Then $(R \oplus R) / K$ is $\tau$-nonsingular. Claim that $(R \oplus R) / K$ is torsion-free $R$-module. For this fact, let us assume $\bar{m} \cdot r=\overline{0}$ and $r \neq 0$ for $\bar{m} \in(R \oplus R) / K$ and $r \in R$. Here $0 \neq r \in$ $\operatorname{Ann}(\bar{m})$. Thus $\operatorname{Ann}(\bar{m}) \neq 0$. Since also $R$ is a commutative domain, then all non-zero ideals of $R$ are essential $25,7.6]$. Thus $A n n(\bar{m})$ is essential ideal in $R$. By hypothesis of the theorem, $\operatorname{Ann}(\bar{m})$ is $\tau$-dense in $R$. Thus $\operatorname{Ann}(\bar{m}) \leq_{\tau_{e}} R$ and so, $\bar{m} \in Z_{\tau}((R \oplus R) / K)$. In this case, $\bar{m}=0$ since $(R \oplus R) / K$ is $\tau$-nonsingular. Therefore $(R \oplus R) / K$ is torsion-free. Thus applying [25, Collary 2.31] $(R \oplus R) / K$ is projective since $(R \oplus R) / K$ is 2-generated over the Prüfer domain $R$. So $(R \oplus R) / K$ is flat by [26, Proposition 3.46]. Thus $K$ is pure in $R \oplus R$ by [17, Proposition 1.3]. Hence $R \oplus R$ is a purely $\tau_{s}$-extending module

$(1) \Rightarrow(9)$ is also similar to $(1) \Rightarrow(5)$. This completes the proof.

In fact, the proof can be also completed by the following implications.

$(4) \Rightarrow(5)$. Let $K$ be a $\tau_{s}$-closed submodule of $R \oplus R$. Then $(R \oplus R) / K$ is $\tau$-nonsingular, i.e., $Z_{\tau}((R \oplus R) / K)=0$. By assumption, since $R$ is a ring with essential ideal of $R$ is $\tau$-dense in it, $\tau$-nonsingular and nonsingular modules are coincide. Therefore $(R \oplus R) / K$ is nonsingular and so $K$ is $s$-closed in $R \oplus R$. By hypothesis, $K$ is pure in $R \oplus R$. Therefore, $R \oplus R$ is purely $\tau_{s}$-extending module.

$(8) \Rightarrow(9)$ is also similar to $(4) \Rightarrow(5)$.

Author Contribution Statements The authors contributed equally and they read and approved the final copy of the manuscript.

Declaration of Competing Interests The authors declare that they have no competing interest.

Acknowledgment The authors wish to express deep appreciation to the referee for his/her valuable comments and suggestions which improved the presentation of this work. 


\section{REFERENCES}

[1] Al-Bahrani, B. H., On purely y-extending modules, Iraqi Journal of Science, 54(3) (2013), $672-675$.

[2] El-Bast, Z. Abd, Smith, P. F., Multiplication modules, Comm. In Algebra, 16(4) (1988), 755-779. https://doi.org/10.1080/00927878808823601

[3] Anderson, F. W., Fuller, K. R., Rings and Categories of Modules. Graduate Texts in Math., No:13, Springer Verlag, New York, 1974. https://doi.org/10.1007/978-1-4612-4418-9

[4] Asgari, Sh., Haghany, A., T-extending modules and $t$-Baer modules, Communications in Algebra, 39(5) (2011), 1605-1623. https://doi.org/10.1080/00927871003677519

[5] Barnard, A., Multiplication modules, Journal of Algebra, 71 (1981), 174-178. https://doi.org/10.1016/0021-8693(81)90112-5

[6] Berktaş, M. K., Doğruöz, S., Tarhan, A., Pure closed subobjects and pure quotient Goldie dimension, JP Journal of Algebra, Number Theory and Applications, 41(1) (2019), 49-57. https://doi.org/10.17654/NT041010049

[7] Chatters, A. W., Hajarnavis, C. R., Rings in which every complement right ideal is a direct summand, Quart. J. Math. Oxford, 28(1) (1977), 61-80. https://doi.org/10.1093/qmath/28.1.61

[8] Clark, J., On purely extending modules, The Proceedings of the International Conference in Abelian Groups and Modules, (1999), 353-358. https://doi.org/10.1007/978-3-0348-7591-2-29

[9] Clark, J., Lomp, C., Vanaja, N., Wisbauer, R., Lifting Modules, Frontiers in Mathematics, Birkhäuser Verlag, Basel, 2006. https://doi.org/10.1007/3-7643-7573-6

[10] Cohn, P. M., On the free product of associative rings, Math. Zeitchr. 71 (1959), 380-398. https://doi.org/10.1007/BF01181410

[11] Crivei, S., Relatively extending modules, Algebr. Represent. Theor., 12(2-5) (2009), 319-332. https://doi.org/10.1007/s10468-009-9155-4

[12] Çeken, S., Alkan, M., On $\tau$-extending modules, Mediterranean Journal of Mathematics, 9(1) (2012), 129-142. https://doi.org/10.1007/s00009-010-0096-2

[13] Doğruöz, S., Classes of extending modules associated with a torsion theory, East-West Journal of Mathematics, 8(2) (2006), 163-180.

[14] Doğruöz, S., Harmanc1, A., Smith, P. F., Modules with unique closure relative to a torsion theory I, Canadian Math. Bull., 53(2) (2010), 230-238. https://doi:10.3906/mat-0712-16

[15] Dung, N. V., Huynh, D. V., Smith, P. F., Wisbauer, R., Extending Modules, Longman, Harlow, 1994. https://doi.org/10.1201/9780203756331

[16] Ertaş, N. O., Fully Idempotent and multiplication modules, Palestine Journal of Mathematics, 3 (2014), 432-437.

[17] Fieldhouse, D. J., Purity and Flatness, Ph.D. Thesis, Department of Mathematics McGill University, Montreal, Canada, July 1967.

[18] Fuchs, L., Note on generalized continuous modules, preprint, (1995).

[19] Golan, J. S., Torsion Theories, Longman, New York, 1986.

[20] Gomez Pardo, J. L., Spectral Gabriel topologies and relative singular functors, Comm. Algebra, 13(1) (1985), 21-57. https://doi.org/10.1080/00927878508823147

[21] Goodearl, K. R., Ring Theory, Nonsingular Rings and Modules, Marcel Dekker, New York, 1976.

[22] Goodearl, K. R., Warfield, R. B., An Introduction to Noncommutative Noetherian Rings, Cambridge University Press, 1989. https://doi.org/10.1017/CBO9780511841699

[23] Harmanc1, A., Smith, P. F., Finite direct sum of CS-modules, Houston J. Math., 19(4), (1993), 523-532.

[24] Kamal, M. A., Muller, B. J., Extending modules over commutative domains, Osaka J. Math., 25 (1988), 531-538. 
[25] Lam, T. Y., Lectures on Modules and Rings, Graduate Texts in Mathematics, 189 Springer-Verlag New York, 1999. https://doi.org/10.1007/978-1-4612-0525-8

[26] Rotman, J. J., An Introduction to Homological Algebra, Academic Press, New York, 1979. https://doi.org/ 10.1007/978-0-387-68324-9

[27] Smith, P. F., Modules for which every submodule has a unique closure, Ring Theory Conference, World Scientific, New Jersey, (1993), 302-313.

[28] Strenström, B., Rings of Quotients, Springer-Verlag, 1975.

[29] Tsai, C. T., Report on Injective Modules, Queen's Paper in Pure and Applied Mathematics, No.6, Kingston, Ontario: Queen's University, 1965.

[30] Wisbauer, R., Foundations of Module and Ring Theory, Gordon and Breach, 1991. https://doi.org/10.1201/9780203755532 